\title{
FUZZY POSSIBILITIES AS UPPER PREVISIONS
}

\author{
LUCA BORTOLUSSI \\ Dipartimento di Matematica e Informatica, University of Udine, Via delle Scienze 206, 33100 \\ Udine, Italy. Email: bortolussi@dimi.uniud.it \\ PAOLO VICIG \\ Dipartimento di Matematica Applicata "B. de Finetti", University of Trieste, Piazzale Europa \\ 1, I-34127 Trieste, Italy. Email: paolo.vicig@econ.units.it \\ Received November 2003
}

\begin{abstract}
In this paper we analyze, mainly in a finitary setting, the consistency properties of fuzzy possibilities, interpreting them as instances of upper previsions and applying the basic notions of avoiding sure loss and coherence from the theory of imprecise probabilities. It ensues that fuzzy possibilities always avoid sure loss, but satisfy the stronger coherence condition only in a special case. Their natural extension, i.e. their least-committal correction to a coherent upper prevision, is determined. The same analysis is then performed when min is replaced by a T-norm (or seminorm) in the definition of fuzzy possibility, showing that the consistency properties and also the natural extension remain the same. Some "closure" properties are also discussed, which are guaranteed to hold if the T-norm is continuous, and are satisfied by (ordinary) possibilities too.
\end{abstract}

Keywords: fuzzy events, fuzzy possibilities, upper previsions, imprecise probabilities.

\section{Introduction}

Real world decisions often happen to be taken about facts which are not quite well specified. Consider, for instance, an investor who decides to buy (or to sell) a financial asset at a certain price because he is told that, very likely, the asset will guarantee (or will not guarantee) a good yield in one year's time.

We pinpoint three facts about this example:

(a) the sentence $S$, "the asset will guarantee a good yield in one year's time", does not represent an event, because of its linguistic indeterminacy, due to the word "good";

(b) the uncertainty evaluation of $S$ is quite rough: $S$ appears to be very likely;

(c) in spite of the intrinsic vagueness of points (a) and (b), the investor may be willing to pay a (non vague, but) certain price to buy the asset.

Since decisional situations similar to the investor example do occur in practice, it is important to study some modelisation which may reasonably closely interpret them. 
Fuzzy set theory provides a conceptual framework for (a): given a partition (or universe of discourse) $\Omega$ (i.e. a set of pairwise disjoint events, whose union is the sure event), a map $F: \Omega \rightarrow[0,1]$ measures the degree of compatibility of each element of $\Omega$ with the concept represented by $S$. Clearly $\Omega$ should be related to $S$, usually because its elements are described by propositions of (classical) logic which solve in alternative ways the indeterminacy in $S$. In our example, one such proposition could be "the asset guarantees an annual gain strictly between $3 \%$ and $4 \%$ of its buying price". The map $F$ identifies the fuzzy event $S$, and is also commonly called fuzzy event. We shall often follow this convention too.

If further a (normal) possibility distribution $\pi$ is given on $\Omega$, a way of tackling (b) is to measure the uncertainty of $S$ (or $F$ ) by the fuzzy possibility $\Pi_{f}$ defined, following Zadeh ${ }^{24}$, as

$$
\Pi_{f}(F)=\sup _{\omega \in \Omega}\{\min \{\pi(\omega), F(\omega)\}\} .
$$

As for point (c), fuzzy set theory does not relate $\Pi_{f}(F)$ with the asset buying or selling price, not explaining if and how a given value of $\Pi_{f}(F)$ determines it, or more generally the investor's behavior.

Behavioral interpretations of uncertainty evaluations are on the contrary welldeveloped in the theory of imprecise probabilities ${ }^{16}$, where the consistency notions of avoiding sure loss (weaker) and coherence (stronger) are applied to upper (and lower) previsions. We shall say more about this approach in section 2.

One link between the two theories is the fact that when $F \in\{0,1\}, F$ is the membership function (or indicator) of an (ordinary) event in the powerset $\mathcal{P}(\Omega)$ of $\Omega$, and $\Pi_{f}(F)=\Pi(F)$ is a possibility - mathematically, a supremum preserving function. A possibility $\Pi$ is a special instance of upper prevision, and is coherent if and only if its restriction on $\Omega$, the possibility distribution $\pi=\left.\Pi\right|_{\Omega}$, is normal, which means $\sup _{\omega \in \Omega}\{\pi(\omega)\}=1^{4, \text { ? }}$. Normal possibilities have several interesting properties within the theory of imprecise probabilities ${ }^{17, ?, ?}$ in particular we shall be concerned in section 3 with some less known closure properties of theirs, i.e. such that they are obtained from properties of coherent upper previsions replacing "coherent upper prevision" with "possibility" in their statement.

In this paper we analyze the behavioral interpretation of fuzzy possibilities as upper previsions in a finitary setting, i.e. referring to a finite partition $\Omega$, showing that they avoid sure loss but are not coherent, apart from a special case, and determining their natural extension, i.e. their least-committal correction to a coherent upper prevision. This is done in section 4 , while in section 5 we answer the same questions for a generalization of (1), called fuzzy T-possibility, where min is replaced by a $\mathrm{T}$-norm (or more generally by a seminorm). Interestingly, it turns out that the generalization does not affect the natural extension, which remains the same (cf. theorem 4 (c)). Also, fuzzy possibilities have the closure properties of possibilities, and this fact seems to depend on the continuity of min. In fact, we show that fuzzy $\mathrm{T}$-possibilities have these properties when $T$ is a continuous norm, 
not necessarily otherwise.

The following interpretation of fuzzy possibilities and T-possibilities is also relevant and will be exploited in the paper: since a map $F: \Omega \rightarrow[0,1]$ corresponds, in probabilistic language, to a random variable (or gamble, according to Walley ${ }^{16}$ ), given a possibility $\Pi$ on $\mathcal{P}(\Omega)$ and considering the set $\mathcal{F}=\mathcal{F}(\Omega)$ of all random variables in $[0,1],(1)$ or also any fuzzy $\mathrm{T}$-possibility are special types of extensions of $\Pi$ onto $\mathcal{F}$. More specifically, they are extensions by means of fuzzy integrals. The question of extending coherent upper probabilities using fuzzy integrals was tackled also by de Cooman ${ }^{2, ?}$. We say more on this in the later section 5.1. Section 6 contains concluding remarks.

\section{Preliminaries}

This section is mainly a concise presentation of some aspects of the theory of imprecise probabilities. A much more extended discussion is in a book of Walley ${ }^{16}$, who made also a shorter on-line presentation ${ }^{18}$.

Although the term "imprecise probabilities" is currently prevailing, the theory in Walley's book ${ }^{16}$ actually deals with imprecise (upper or lower) previsions, which are (imprecise) uncertainty evaluations for bounded random variables. An imprecise probability for an event $A$ is a special case of upper prevision where the random variable is the indicator of $A, I(A)$, i.e. the random variable which is 1 when $A$ is true, 0 when $A$ is false.

In Walley's book ${ }^{16}$, following and extending an approach which goes back to de Finetti ${ }^{7}$, imprecise previsions are given a behavioral interpretation in terms of betting schemes. The upper (lower) prevision $\bar{P}(X)(\underline{P}(X))$ an agent assigns to a (bounded) random number $X$ is his/her infimum selling price (supremum buying price) for $X$. Since selling $X$ is equivalent to buying $-X$, we may focus on upper previsions only $(\bar{P}(X)=-\underline{P}(-X))$.

In other words, given $\bar{P}(X)$, the agent is willing to accept the bet which (as he receives at least $\bar{P}(X)$ for selling $X)$ guarantees him the uncertain gain $\bar{G}(X)=$ $\bar{P}(X)-X$, which might possibly be negative for some values of $X$. It is however unreasonable that $\sup \bar{G}<0$, because this would cause a sure loss to the agent, if the selling price is sufficiently close to $\bar{P}(X)$. Further, if the agent finds $\bar{P}(X)-X$ acceptable, and has a linear utility scale, he should find $s(\bar{P}(X)-X)$ acceptable too, $\forall s \geq 0$.

A generalization of this idea leads to the consistency notion of avoiding sure loss for an upper prevision $\bar{P}: \mathcal{D} \rightarrow \mathbb{R}$, where $\mathcal{D}$ is an arbitrary set of bounded random numbers.

Definition 1. Given $\bar{P}: \mathcal{D} \rightarrow \mathbb{R}, \bar{P}$ is an upper prevision that avoids sure loss if and only if $\forall n \in \mathbb{N}^{+}, \forall s_{1}, \ldots, s_{n} \geq 0, \forall X_{1}, \ldots, X_{n} \in \mathcal{D}$, defining $\bar{G}=\sum_{i=1}^{n} s_{i}\left(\bar{P}\left(X_{i}\right)-\right.$ $\left.X_{i}\right), \sup \bar{G} \geq 0$.

Definition 1 is a natural consistency requirement: if $\bar{P}$ does not avoid sure loss, 
there exists a finite set of bets, all individually acceptable, giving an overall sure loss.

However, avoiding sure loss is too mild a consistency notion. For instance, it does not necessarily require $\bar{P}(X) \leq \sup X$, nor does it require monotonicity. Although special cases of previsions that avoid sure loss, with certain additional properties, may correspond to interesting models ${ }^{11}$, the stronger coherence condition is usually preferable.

Definition 2. Given $\bar{P}: \mathcal{D} \rightarrow \mathbb{R}, \bar{P}$ is a coherent upper prevision if and only if $\forall n \in \mathbb{N}, \forall s_{0}, \ldots, s_{n} \geq 0, \forall X_{0}, \ldots, X_{n} \in \mathcal{D}$, defining $\bar{G}=\sum_{i=1}^{n} s_{i}\left(\bar{P}\left(X_{i}\right)-X_{i}\right)-$ $s_{0}\left(\bar{P}\left(X_{0}\right)-X_{0}\right), \sup \bar{G} \geq 0$.

We refer to Walley's book ${ }^{16}$ for a behavioral interpretation of coherence. Possibility measures, plausibility functions, 2-alternating probabilities are special cases of coherent upper (probabilities, and hence) previsions ${ }^{16, ?}$.

A coherent precise prevision $P$ on $\mathcal{D}$ is a map $P: \mathcal{D} \rightarrow \mathbb{R}$ that satisfies the modified version of definition 1 where " $s_{1}, \ldots, s_{n} \geq 0$ " is replaced by " $s_{1}, \ldots, s_{n} \in \mathbb{R}$ " 7 , and is a special case of both upper and lower coherent prevision ${ }^{16}$.

Remark 1. Although its definition does not involve any probability distribution, the notion of precise prevision is equivalent to that of expectation (a proof may be found in a report of Crisma ${ }^{6}$ ).

In particular, we shall be concerned in theorem 3 with precise previsions on the set $\mathcal{F}$ of all mappings from $\Omega=\left\{\omega_{1}, \ldots, \omega_{k}\right\}$ into $[0,1]$. Since, for $i=1, \ldots, k$, the indicator function $I\left(\omega_{i}\right)$ of $\omega_{i}$ belongs to $\mathcal{F}$, and its prevision $P\left(I\left(\omega_{i}\right)\right)$ is simply the probability of $\omega_{i}$, any precise prevision $P$ on $\mathcal{F}$ uniquely determines a probability on $\Omega$, i.e. a probability vector $\left(p_{1}, \ldots, p_{k}\right)$ such that $p_{i}=P\left(I\left(\omega_{i}\right)\right)$ is the probability of $\omega_{i}$. Conversely, given a probability vector $\left(p_{1}, \ldots, p_{k}\right)$ on $\Omega$, a coherent precise prevision $P$ on $\mathcal{F}$ is determined by computing, $\forall F \in \mathcal{F}$, its expectation $E(F)=$ $\sum_{i=1}^{k} p_{i} F\left(\omega_{i}\right)$ and putting $P(F)=E(F)$. Clearly, $P$ extends $\left(p_{1}, \ldots, p_{k}\right)$ on $\mathcal{F}$, and is further its unique extension on $\mathcal{F}$ to a coherent precise prevision.

Previsions that avoid sure loss and coherent previsions are characterized indirectly using precise previsions:

Theorem 1. Given $\bar{P}: \mathcal{D} \rightarrow \mathbb{R}$,

(a) $\bar{P}$ is an upper prevision that avoids sure loss if and only if $\bar{P}$ dominates a coherent precise prevision $P$ on $\mathcal{D}$, i.e. iff $\bar{P}(X) \geq P(X), \forall X \in \mathcal{D}$;

(b) (Upper envelope theorem) $\bar{P}$ is a coherent upper prevision if and only if $\bar{P}(X)=$ $\sup _{P \in \mathcal{M}}\{P(X)\}, \forall X \in \mathcal{D}$, where $\mathcal{M}$ is some non-empty set of coherent precise previsions (sup is attained). $\bar{P}$ is called the upper envelope of $\mathcal{M}$.

Another fundamental concept in the theory is that of natural extension, defined in Walley's book ${ }^{16}$. In our framework, it is sufficient to recall that the natural extension $\bar{E}$ on $\mathcal{D}$ of an upper prevision which avoids sure loss on $\mathcal{D}$ (is always 
finite and) is its least-committal correction to a coherent upper prevision on $\mathcal{D}$. This follows from (c) in the next theorem, which collects some properties of the natural extension to be used later.

Theorem 2. Given $\bar{P}: \mathcal{D} \rightarrow \mathbb{R}$, suppose that $\bar{P}$ avoids sure loss and let $\mathcal{M}^{*}$ be the set of all coherent precise probabilities dominated by $\bar{P}$ on $\mathcal{D}$. Then

(a) the natural extension $\bar{E}$ of $\bar{P}$ is given by $\bar{E}(X)=\max _{P \in \mathcal{M}^{*}}\{P(X)\}, \forall X \in \mathcal{D}$, and $\mathcal{M}^{*}(\bar{P})=\mathcal{M}^{*}(\bar{E})$;

(b) $\bar{P}$ is coherent if and only if $\bar{P}=\bar{E}$;

(c) if $\bar{P}^{*}$ is a coherent upper prevision dominated by $\bar{P}$, then $\bar{P}^{*}(X) \leq \bar{E}(X)$, $\forall X \in \mathcal{D}$.

Note in particular that (a) is a characterization of the natural extension in Walley's book ${ }^{16}$, but can be taken as its definition in our framework.

\section{Closure properties of possibilities}

We recall that given a (not necessarily finite) partition $\Omega, \Pi: \mathcal{P}(\Omega) \rightarrow[0,1]$ is a possibility (measure) if there exists a function $\pi: \Omega \rightarrow[0,1]$ (called possibility distribution) such that

$$
\Pi(A)=\sup _{\omega \in A}\{\pi(\omega)\}, \forall A \in \mathcal{P}(\Omega)
$$

(assuming $\Pi(\emptyset)=0$ ); hence $\Pi$ is a supremum preserving function. Further, $\Pi$ is normal if and only if $\sup _{\omega \in \Omega}\{\pi(\omega)\}=1$. In the sequel we shall consider normal possibilities only.

Possibility measures have been studied in a number of different theories, in particular fuzzy set theory (some general references include the books of Dubois and Prade $^{8}$ and Wang and $\mathrm{Klir}^{20}$ ), and, more recently, imprecise probability theory.

Within the theory of imprecise probabilities, a possibility is viewed as an instance of upper prevision, and is coherent if and only if it is normal ${ }^{4, ?}$; non-normal possibilities incur sure loss, as is easy to verify.

Although a possibility is a rather special case of upper prevision, when viewed as upper previsions possibilities have several interesting features, investigated by de Cooman, Walley and others, $2, ?, ?, ?, ?, ?$.

In particular, we shall be concerned in this section with a further aspect of possibilities, that is their complying with a certain number of "closure" properties. This means properties of coherent upper previsions which hold also when replacing "coherent upper prevision" with "possibility" in their statement. For instance, an upper envelope of possibilities is a coherent upper prevision, by a general result in Walley's book ${ }^{16}$ about upper envelopes of coherent upper previsions (and hence of possibilities), but it is also a possibility by the next proposition. The subsequent propositions concern uniform and pointwise convergence of sequences of possibilities. One motivation for studying such properties is that, as we shall see in later 
sections, they are to a large extent preserved when extending possibilities to fuzzy possibilities and fuzzy T-possibilities.

Proposition 1. Let $\left\{\Pi_{\gamma}\right\}_{\gamma \in \Gamma}$ be a set of possibility measures on $\mathcal{P}(\Omega)$. Its upper envelope $\Pi^{*}$, defined by $\Pi^{*}(A)=\sup _{\gamma \in \Gamma}\left\{\Pi_{\gamma}(A)\right\}, \forall A \in \mathcal{P}(\Omega)$, is a possibility measure on $\mathcal{P}(\Omega)$.

Proof. Follows easily from supremum preserving properties of possibility measures, cf. (2).

Proposition 2. Let $\left\{\Pi_{n}\right\}_{n \in \mathbb{N}^{+}}$be a sequence of possibility measures on $\mathcal{P}(\Omega)$, where $\Omega$ is not necessarily finite, that converges uniformly to a function $\Pi$. Then $\Pi$ is a possibility measure.

Proof. We prove first that the limit function $\pi$ of the sequence $\left\{\pi_{n}\right\}\left(\pi_{n}\right.$ is the possibility distribution of $\Pi_{n}$ ) is a possibility distribution. In fact, since $0 \leq \pi_{n}(\omega) \leq$ $1, \forall n \in \mathbb{N}^{+}, \pi$ is non-negative and $\sup _{\omega \in \Omega}\{\pi(\omega)\} \leq 1$. We show that actually $\sup _{\omega \in \Omega}\{\pi(\omega)\}=1$. Since the convergence of $\left\{\pi_{n}\right\}$ is uniform,

$$
\forall \varepsilon>0, \exists \bar{n} \text { such that } \forall n \geq \bar{n}, \sup _{\omega \in \Omega}\left|\pi_{n}(\omega)-\pi(\omega)\right|<\varepsilon
$$

Therefore the following inequalities hold for $n \geq \bar{n}$ (suprema are performed over all $\omega \in \Omega)$

$$
\varepsilon>\sup \left|\pi_{n}(\omega)-\pi(\omega)\right| \geq \sup \left\{\pi_{n}(\omega)-\pi(\omega)\right\} \geq \sup \left\{\pi_{n}(\omega)\right\}-\sup \{\pi(\omega)\},
$$

from which $\sup \{\pi(\omega)\} \geq \sup \left\{\pi_{n}(\omega)\right\}-\varepsilon=1-\varepsilon$. Summing up,

$$
\forall \varepsilon>0,1-\varepsilon<\sup \{\pi(\omega)\} \leq 1, \text { i.e. } \sup \{\pi(\omega)\}=1 .
$$

To complete the proof, we have to show that (2) holds. In fact, $\Pi_{n}(A) \rightarrow \sup _{\omega \in A}\{\pi(\omega)\}$ and its convergence is uniform, because

$$
\begin{array}{r}
\sup _{A \in \mathcal{P}(\Omega)}\left|\Pi_{n}(A)-\sup _{\omega \in A}\{\pi(\omega)\}\right|=\sup _{A \in \mathcal{P}(\Omega)}\left|\sup _{\omega \in A}\left\{\pi_{n}(\omega)\right\}-\sup _{\omega \in A}\{\pi(\omega)\}\right| \\
\leq \sup _{A \in \mathcal{P}(\Omega)}\left|\sup _{\omega \in A}\left\{\pi_{n}(\omega)-\pi(\omega)\right\}\right| \leq \sup _{A \in \mathcal{P}(\Omega)} \sup _{\omega \in A}\left|\pi_{n}(\omega)-\pi(\omega)\right| \\
\leq \sup _{A \in \mathcal{P}(\Omega)} \sup _{\omega \in \Omega}\left|\pi_{n}(\omega)-\pi(\omega)\right| \leq n \geq \bar{n} \sup _{A \in \mathcal{P}(\Omega)} \varepsilon=\varepsilon
\end{array}
$$

where the last inequality follows from the uniform convergence of $\left\{\pi_{n}\right\}$ to $\pi$, that is from (3).

On the other hand, from the theorem assumptions $\Pi_{n}(A) \rightarrow \Pi(A), \forall A \in \mathcal{P}(\Omega)$. Hence, (2) follows from uniqueness of the limit.

Proposition 3. (Pointwise convergence) Let $\left\{\Pi_{n}\right\}_{n \in \mathbb{N}^{+}}$be a sequence of possibility measures on $\mathcal{P}(\Omega)$ which converges pointwise to a function $\Pi$ on $\mathcal{P}(\Omega)$, i.e. $\Pi_{n}(A) \rightarrow \Pi(A), \forall A \in \mathcal{P}(\Omega)$. 
Then $\Pi$ is a possibility measure if $\Omega$ is finite, while this is not necessarily true when $\Omega$ is infinite.

Proof. If $\Omega$ is finite, the assumption follows from proposition 2, recalling that uniform and pointwise convergence are then equivalent.

We give now an example where $\Omega$ is infinite and $\Pi$ is no possibility: let $\Omega=$ $\left\{\omega_{1}, \ldots, \omega_{j}, \ldots\right\}$ and consider the sequence of $0-1$ valued possibility distributions $\left\{\pi_{n}\right\}_{n \in \mathbb{N}^{+}}$, where $\pi_{n}\left(\omega_{j}\right)=1$ if $j=n, \pi_{n}\left(\omega_{j}\right)=0$ if $j \neq n$.

The sequence of possibility measures $\left\{\Pi_{n}\right\}$ induced by $\left\{\pi_{n}\right\}$ converges pointwise to a limit function $\Pi$ which is no possibility, because (2) does not hold. In fact $1=\Pi(\Omega)>\sup _{j \in \mathbb{N}^{+}}\left\{\pi\left(\omega_{j}\right)\right\}=0$, where $\pi=\lim _{n \rightarrow \infty} \pi_{n}$.

To end this section, we note that possibilities are not closed with respect to some other properties of coherent upper previsions. For instance, while a convex combination of coherent upper previsions is a coherent upper prevision ${ }^{16}$, it is easy to find examples of convex combinations of possibilities which are not possibilities.

\section{Fuzzy possibilities as upper previsions}

In this section we investigate the consistency properties of fuzzy possibilities as imprecise previsions. Recall from section 1 that $\mathcal{F}$ is the set of all maps $F$ from a given partition $\Omega$ into $[0,1]$. Moreover, whenever $F \in\{0,1\}, F$ is the indicator function of some event $A$ in the power set of $\Omega$, and (1) reduces to the possibility of $A, \Pi(A)$, hence to a coherent upper prevision (probability) for $A$. It ensues that a fuzzy possibility may be interpreted as (an extension of) an upper prevision. Its consistency properties are investigated in section 4.1, where $\Omega$ is a finite partition. This assumption does not necessarily hold in section 4.2 , where we study closure properties of fuzzy possibilities.

\subsection{Fuzzy possibilities, coherence, and natural extension}

Since $\Omega=\left\{\omega_{1}, \ldots, \omega_{k}\right\}$ in this section, (1) reduces to

$$
\Pi_{f}(F)=\max _{\omega \in \Omega}\{\min \{\pi(\omega), F(\omega)\}\} .
$$

The basic result for analyzing consistency of $\Pi_{f}$ is the following theorem, which characterizes the set of (coherent) precise previsions dominated by $\Pi_{f}$.

Theorem 3. Let $\Pi_{f}$ be a fuzzy possibility, defined by (4). Define

$$
I_{\Pi}=\left\{i \in\{1, \ldots, k\} \mid \pi_{i}=1\right\} .
$$

The set $\mathcal{M}\left(\Pi_{f}\right)$ of coherent precise previsions dominated by $\Pi_{f}$ on $\mathcal{F}$ is characterized as follows:

$$
\mathcal{M}\left(\Pi_{f}\right)=\left\{P \mid P\left(I\left(\omega_{i}\right)\right)>0 \Leftrightarrow i \in I_{\Pi}\right\} .
$$


Proof. We preliminarily recall (cf. Remark 1) that a (coherent) precise prevision $P$ on $\mathcal{F}$ is uniquely identified by a probability vector $\left(p_{1}, \ldots, p_{k}\right)$ on $\Omega$, so that we can write $p_{i}=P\left(I\left(\omega_{i}\right)\right)$.

Given this, consider $P$ such that $p_{i}=P\left(I\left(\omega_{i}\right)\right)>0$ if and only if $i \in I_{\Pi}$ (there are such precise previsions, because normality of $\pi$ guarantees that there exists $j \in\{1, \ldots, k\}$ such that $\pi\left(\omega_{j}\right)=1$, i.e. $I_{\Pi}$ is non-empty).

We show that $P$ is dominated by $\Pi_{f}(F)$, and therefore belongs to $\mathcal{M}\left(\Pi_{f}\right)$. In fact, $\forall i \in I_{\Pi}, \min \left\{\pi_{i}, F\left(\omega_{i}\right)\right\}=F\left(\omega_{i}\right)$, hence $\Pi_{f}(F) \geq \max _{I_{\Pi}}\left\{F\left(\omega_{i}\right)\right\}$. It ensues that, $\forall F \in \mathcal{F}$,

$$
P(F)=\sum_{i=1}^{k} p_{i} F\left(\omega_{i}\right)=\sum_{I_{\Pi}} p_{i} F\left(\omega_{i}\right) \leq \sum_{I_{\Pi}} p_{i} \Pi_{f}(F)=\Pi_{f}(F) .
$$

Conversely, let $P$ be a precise prevision such that, for some $j, p_{j}=P\left(I\left(\omega_{j}\right)\right)>0$ and $j \notin I_{\Pi}$. Then $P$ does not belong to $\mathcal{M}\left(\Pi_{f}\right)$.

To see this, suppose for notational ease that $\pi\left(\omega_{1}\right)=1, \pi\left(\omega_{2}\right)=\pi_{2}<1$, and $p_{2}>0$. Consider $F \in \mathcal{F}$ such that $F\left(\omega_{2}\right)=1$ and $F\left(\omega_{i}\right)=\pi_{2}$ for $i \neq 2$. Since $\min \left\{\pi\left(\omega_{1}\right), F\left(\omega_{1}\right)\right\}=\min \left\{\pi\left(\omega_{2}\right), F\left(\omega_{2}\right)\right\}=\pi_{2}$ and $\min \left\{\pi\left(\omega_{i}\right), F\left(\omega_{i}\right)\right\} \leq \pi_{2}$ for $i>2$, we obtain $\Pi_{f}(F)=\pi_{2}$. Then

$$
\begin{aligned}
P(F)=\sum_{i=1}^{k} p_{i} F\left(\omega_{i}\right) & =p_{2}+\sum_{i \neq 2} p_{i} \pi_{2}=p_{2}+\pi_{2}\left(1-p_{2}\right) \\
& =p_{2}\left(1-\pi_{2}\right)+\pi_{2}>\pi_{2}=\Pi_{f}(F) .
\end{aligned}
$$

We may thus state that the set $\mathcal{M}\left(\Pi_{f}\right)$ is characterized by (6).

Corollary 1. If $\pi$ is unimodal, the cardinality of $\mathcal{M}\left(\Pi_{f}\right)$ is one.

We shall also need the following definition, before turning to consistency properties of $\Pi_{f}$.

Definition 3. A fuzzy possibility $\Pi_{f}$ is termed non-comparative if and only if it is generated by a $\{0,1\}$-valued possibility distribution, is termed comparative otherwise.

Proposition 4. Let $\Pi_{f}$ be a fuzzy possibility. Then $\Pi_{f}$ avoids sure loss. Moreover, $\Pi_{f}$ is coherent if and only if it is non-comparative.

Proof. It was shown in the proof of Theorem 3 that $\mathcal{M}\left(\Pi_{f}\right)$ is non-empty. Therefore $\Pi_{f}$ avoids sure loss by theorem 1 (a).

To prove the remaining part of the theorem, we prove (equivalently, by theorem 1 (b)) that $\Pi_{f}$ is the upper envelope of the set $\mathcal{M}\left(\Pi_{f}\right)$ if and only if it is noncomparative.

Let $\Pi_{f}$ be non-comparative. Then $\Pi_{f}(F)=\max _{i \in I_{\Pi}}\left\{F\left(\omega_{i}\right)\right\}$.

Now let $F \in \mathcal{F}$, and suppose that $\Pi_{f}(F)=F\left(\omega_{j}\right)$. The precise prevision $P^{*}$ generated by the probability vector $\left(p_{1}, \ldots, p_{k}\right)$ with $p_{j}=1, p_{i}=0$ for $i \neq j$ 
(belongs to $\mathcal{M}\left(\Pi_{f}\right)$ and) is such that $P^{*}(F)=F\left(\omega_{j}\right)=\Pi_{f}(F)$. Clearly, any other prevision in $\mathcal{M}\left(\Pi_{f}\right)$ is dominated by $\Pi_{f}$ by theorem 3 . Therefore $\Pi_{f}$ is the upper envelope of $\mathcal{M}\left(\Pi_{f}\right)$.

Vice versa, let $\Pi_{f}$ be a comparative fuzzy possibility. Hence there exists $\pi_{j}=$ $\pi\left(\omega_{j}\right)$ such that $0<\pi_{j}<1$. Let $0 \leq f<\pi_{j}$, and consider the following $F^{*} \in \mathcal{F}$ :

$$
F^{*}\left(\omega_{i}\right)=\left\{\begin{array}{l}
f \text { if } i \in I_{\Pi} \\
\pi_{j} \text { if } i=j \\
0 \text { otherwise }
\end{array}\right.
$$

Then $\Pi_{f}\left(F^{*}\right)=\pi_{j}>f$, but if $P$ is any precise prevision in $\mathcal{M}\left(\Pi_{f}\right)$,

$$
P\left(F^{*}\right)=\sum_{i \in I_{\Pi}} p_{i} F^{*}\left(\omega_{i}\right)=\sum_{i \in I_{\Pi}} p_{i} f=f<\pi_{j}=\Pi_{f}\left(F^{*}\right),
$$

from which follows that $\Pi_{f}$ is not the upper envelope of $\mathcal{M}\left(\Pi_{f}\right)$ on $\mathcal{F}$, hence it is not coherent.

Proposition 5. (Natural extension theorem) Let $\Pi_{f}: \mathcal{F} \rightarrow[0,1]$ be a fuzzy possibility on $\Omega$. The natural extension of $\Pi_{f}$ on $\mathcal{F}$ is

$$
\bar{E}_{\Pi_{f}}(F)=\max _{i \in I_{\Pi}}\left\{F\left(\omega_{i}\right)\right\}, \forall F \in \mathcal{F}
$$

Proof. By theorem 2 (a) it is sufficient to show that $\bar{E}_{\Pi_{f}}(F)$ is the upper envelope of the set $\mathcal{M}\left(\Pi_{f}\right)$. To do that, choose $F \in \mathcal{F}$. Whatever is $P \in \mathcal{M}\left(\Pi_{f}\right)$,

$$
P(F)=\sum_{i \in I_{\Pi}} p_{i} F\left(\omega_{i}\right) \leq \sum_{i \in I_{\Pi}} p_{i} \max _{i \in I_{\Pi}}\left\{F\left(\omega_{i}\right)\right\}=\max _{i \in I_{\Pi}}\left\{F\left(\omega_{i}\right)\right\} .
$$

Further, equality is achieved above for some $P \in \mathcal{M}\left(\Pi_{f}\right)$. In fact, let $\max _{i \in I_{\Pi}}\left\{F\left(\omega_{i}\right)\right\}=F\left(\omega_{j}\right)$ and consider the precise prevision $P^{*}$ generated by the probability vector $\left(p_{1}, \ldots, p_{k}\right)$, where $p_{j}=1, p_{i}=0$ for $i \neq j$.

Then $P^{*}$ belongs to $\mathcal{M}\left(\Pi_{f}\right)$, and $P^{*}(F)=\sum_{i \in I_{\Pi}} p_{i} F\left(\omega_{i}\right)=F\left(\omega_{j}\right)$.

Comment. A fuzzy possibility is generally not coherent, but avoids sure loss. Its natural extension $\bar{E}$ has a rather simple expression. Loosely speaking, $\bar{E}$ is a sort of "defuzzification", as it takes account only of those events in $\Omega$ which have possibility one (i.e., the most likely ones), and among them chooses the one(s) most compatible with $F$ (having the maximum $F(\cdot)$ ).

\subsection{Some properties of fuzzy possibilities}

Fuzzy possibilities, although being generally not coherent, preserve however some closure properties of possibilities, as shown in the following proposition.

Proposition 6. (a) Let $\mathcal{P}_{\Gamma}=\left\{\Pi_{f_{\gamma}}\right\}_{\gamma \in \Gamma}$ be a family of fuzzy possibilities on $\mathcal{F}=\mathcal{F}(\Omega)$, where $\Omega$ is arbitrary. The upper envelope $\Pi_{f}{ }^{*}$ of $\mathcal{P}_{\Gamma}, \Pi_{f}{ }^{*}(F)=$ $\sup _{\gamma \in \Gamma}\left\{\Pi_{f_{\gamma}}(F)\right\}, \forall F \in \mathcal{F}$, is a fuzzy possibility. 
(b) Let $\Omega$ be finite and let $\left\{\Pi_{f}^{n}\right\}_{n \in \mathbb{N}^{+}}$be a sequence of fuzzy possibilities on $\mathcal{F}(\Omega)$ which converges pointwise to $\Pi_{f}$ on $\mathcal{F}$, i.e. $\Pi_{f}^{n}(F) \rightarrow \Pi_{f}(F), \forall F \in \mathcal{F}$. Then $\Pi_{f}$ is a fuzzy possibility.

Proof. We preliminarily observe that the restriction $\left.\Pi_{f}\right|_{\mathcal{F}_{0,1}}$ of a fuzzy possibility $\Pi_{f}$ to the set $\mathcal{F}_{0,1}$ of all binary $(0-1$ valued) $F \in \mathcal{F}$ is an ordinary possibility on $\mathcal{P}(\Omega)$.

Proof of (a). Since $\left\{\left.\Pi_{f_{\gamma}}\right|_{\mathcal{F}_{0,1}}\right\}_{\gamma \in \Gamma}$ is a family of ordinary possibility measures, its upper envelope is a possibility by proposition 1 . In particular $\Pi_{f}^{*}(\omega)=\pi^{*}(\omega)$ is a possibility distribution on $\Omega$. Hence we have to prove that

$$
\Pi_{f}^{*}(F)=\sup _{\omega \in \Omega}\left\{\min \left\{\pi^{*}(\omega), F(\omega)\right\}\right\}, \forall F \in \mathcal{F} .
$$

We prove first that

$$
\sup _{\gamma \in \Gamma}\left\{\min \left\{\pi_{\gamma}(\omega), F(\omega)\right\}\right\}=\min \left\{\sup _{\gamma \in \Gamma}\left\{\pi_{\gamma}(\omega)\right\}, F(\omega)\right\} .
$$

To obtain (9), consider a monotone sequence $\pi_{\gamma_{n}}(\omega) \uparrow \sup _{\gamma \in \Gamma}\left\{\pi_{\gamma}(\omega)\right\}$. Then we get, using the sequence's monotonicity at the second equality and continuity of $f(x, y)=\min (x, y), f: \mathbb{R}^{2} \rightarrow \mathbb{R}$, a well known real analysis result (see, for instance, the book of Rudin $\left.{ }^{12}\right)$, at the third equality:

$$
\begin{gathered}
\sup _{\gamma \in \Gamma}\left\{\min \left\{\pi_{\gamma}(\omega), F(\omega)\right\}\right\}=\sup _{\gamma_{n} \in \Gamma}\left\{\min \left\{\pi_{\gamma_{n}}(\omega), F(\omega)\right\}\right\} \\
=\lim _{n \rightarrow \infty}\left\{\min \left\{\pi_{\gamma_{n}}(\omega), F(\omega)\right\}\right\}=\min \left\{\lim _{n \rightarrow \infty} \pi_{\gamma_{n}}(\omega), F(\omega)\right\} \\
=\min \left\{\sup _{\gamma \in \Gamma}\left\{\pi_{\gamma}(\omega)\right\}, F(\omega)\right\} .
\end{gathered}
$$

Using also (9), we obtain then (8) as follows:

$$
\begin{gathered}
\Pi_{f}^{*}(F)=\sup _{\gamma \in \Gamma}\left\{\Pi_{f}(F)\right\}=\sup _{\gamma \in \Gamma} \sup _{\omega \in \Omega}\left\{\min \left\{\pi_{\gamma}(\omega), F(\omega)\right\}\right\} \\
=\sup _{\omega \in \Omega} \sup _{\gamma \in \Gamma}\left\{\min \left\{\pi_{\gamma}(\omega), F(\omega)\right\}\right\}=\sup _{\omega \in \Omega}\left\{\min \left\{\sup _{\gamma \in \Gamma}\left\{\pi_{\gamma}(\omega)\right\}, F(\omega)\right\}\right\} \\
=\sup _{\omega \in \Omega}\left\{\min \left\{\pi^{*}(\omega), F(\omega)\right\}\right\} .
\end{gathered}
$$

Proof of (b). Proposition 3 guarantees that, $\forall F \in \mathcal{F}_{0,1},\left.\Pi_{f}\right|_{\mathcal{F}_{0,1}}=$ $\left.\lim _{n \rightarrow \infty} \Pi_{f}^{n}\right|_{\mathcal{F}_{0,1}}$ is an ordinary possibility measure. Hence $\pi=\lim _{n \rightarrow \infty} \pi_{n}$ is a possibility distribution on $\Omega$. We have to show now that (4) holds for $\Pi_{f}$.

From continuity of minimum and the convergence assumption we get, $\forall \omega \in \Omega$, $\min \left\{\pi_{n}(\omega), F(\omega)\right\} \rightarrow \min \{\pi(\omega), F(\omega)\}$, and using also continuity of maximum

$$
\max _{\omega \in \Omega}\left\{\min \left\{\pi_{n}(\omega), F(\omega)\right\}\right\} \rightarrow \max _{\omega \in \Omega}\{\min \{\pi(\omega), F(\omega)\}\} .
$$

It follows that:

$$
\begin{array}{ccc}
\Pi_{f}^{n}(F)= & \max _{\omega \in \Omega}\left\{\min \left\{\pi_{n}(\omega), F(\omega)\right\}\right\} \\
\downarrow & \downarrow \\
\Pi_{f}(F) & \max _{\omega \in \Omega}\{\min \{\pi(\omega), F(\omega)\}\}
\end{array}
$$

and uniqueness of the limit gives then (4). 


\section{A generalization of fuzzy possibilities}

It is natural in fuzzy set theory to replace the minimum operator with a more general one, often a T-norm (see for instance the book of Nguyen and Walker ${ }^{9}$, or the preentation of Yager and Fodor ${ }^{22}$ ). We shall do that too, considering now a fuzzy T-possibility, defined by

$$
\Pi_{T}(F)=\sup _{\omega \in \Omega}\{T(\pi(\omega), F(\omega))\}
$$

where $T$ is a T-norm, and answering the same questions raised in section 4. Again, we call non-comparative a fuzzy T-possibility generated by a $\{0,1\}$ valued possibility distribution.

Definition 4. We recall that a triangular norm or $T-$ norm is a mapping $T$ : $[0,1]^{2} \rightarrow[0,1]$ such that:

(a) $\forall x \in[0,1], T(x, 1)=x$ (identity)

(b) $\forall x, y \in[0,1], T(x, y)=T(y, x)$ (commutativity)

(c) $\forall x, y, z \in[0,1], T(x, T(y, z))=T(T(x, y), z)$ (associativity)

(d) $\forall x, y, z, w \in[0,1]$, such that $x \leq z$ and $y \leq w, T(x, y) \leq T(z, w)$ (isotonicity or monotonicity),

while a $T$-seminorm ${ }^{1}$ is a mapping $S:[0,1]^{2} \rightarrow[0,1]$ satisfying (d) and (a') $\forall x \in[0,1], S(x, 1)=S(1, x)=x$.

Clearly, every $\mathrm{T}$-norm is also a $\mathrm{T}$-seminorm. Further, it is easy to verify that properties (a'), (d) imply

(e) $\forall x \in[0,1], S(0, x)=S(x, 0)=0$.

Theorem 4. Let $\Pi_{T}: \mathcal{F} \rightarrow[0,1]$ be a fuzzy T-possibility on $\mathcal{F}=\mathcal{F}(\Omega)$, where $\Omega=\left\{\omega_{1}, \ldots, \omega_{k}\right\}$. If $\Pi_{T}$ is comparative, then:

(a) $\Pi_{T}$ is not coherent

(b) $\Pi_{T}$ avoids sure loss

(c) the natural extension $\bar{E}_{\Pi_{T}}$ of $\Pi_{T}$ is, $\forall F \in \mathcal{F}$,

$$
\bar{E}_{\Pi_{T}}(F)=\max _{i \in I_{\Pi}}\left\{F\left(\omega_{i}\right)\right\}=\bar{E}_{\Pi_{f}}(F),
$$

where $I_{\Pi}$ is defined in (5) and $\bar{E}_{\Pi_{f}}$ is the natural extension of $\Pi_{f}$, the fuzzy $T$ possibility with $T=\min$.

Further, if $\Pi_{T}$ is non-comparative it is coherent.

Proof. To prove (a), recall that the set $\mathcal{T}$ of all T-norms has a maximal and a minimal element, which means in our framework that $\forall T \in \mathcal{T}, \forall \omega, \forall(\pi(\omega), F(\omega))$,

$$
Z(\pi(\omega), F(\omega)) \leq T(\pi(\omega), F(\omega)) \leq \min (\pi(\omega), F(\omega)),
$$

where $Z$ is the drastic product defined by

$$
Z(\pi(\omega), F(\omega))= \begin{cases}\min (\pi(\omega), F(\omega)) & \text { if } \max (\pi(\omega), F(\omega))=1 \\ 0 & \text { otherwise }\end{cases}
$$


We prove now that

$$
\bar{E}_{\Pi_{f}}(F) \leq \Pi_{Z}(F),
$$

whatever is the given possibility distribution $\pi$ ( $\Pi_{Z}$ is the fuzzy T-possibility where $T$ is the drastic product $Z$ ).

In fact, putting $I_{F}=\left\{i \in\{1, \ldots, k\} \mid F\left(\omega_{i}\right)=1\right\}$,

$$
\Pi_{Z}(F)=\max _{\omega \in \Omega}\{Z(\pi(\omega), F(\omega))\}=\max _{i \in I_{\Pi} \cup I_{F}}\left\{\min \left\{\pi_{i}, F\left(\omega_{i}\right)\right\}\right\} .
$$

Using also proposition 5 , we obtain now $(14), \forall F \in \mathcal{F}$ :

$$
\begin{gathered}
\bar{E}_{\Pi_{f}}(F)=\max _{i \in I_{\Pi}}\left\{F\left(\omega_{i}\right)\right\}=\max _{i \in I_{\Pi}}\left\{\min \left\{\pi\left(\omega_{i}\right), F\left(\omega_{i}\right)\right\}\right\} \\
\leq \max _{i \in I_{\Pi} \cup I_{F}}\left\{\min \left\{\pi\left(\omega_{i}\right), F\left(\omega_{i}\right)\right\}\right\}=\Pi_{Z}(F) .
\end{gathered}
$$

There exists $F \in \mathcal{F}$ which makes the inequality strict in (14). In fact, since $\Pi_{Z}$ is comparative there is $\omega_{j} \in \Omega$ such that $0<\pi\left(\omega_{j}\right)<1$. Let $F \in \mathcal{F}$ be defined by $F\left(\omega_{j}\right)=1, F\left(\omega_{i}\right)=0, \forall i \neq j$. It is immediate to see that $\Pi_{Z}(F)=\pi\left(\omega_{j}\right)>0=$ $\bar{E}_{\Pi_{f}}(F)$.

Apply now the maximum operator in (12) and use (14) to get

$$
\bar{E}_{\Pi_{f}}(F) \leq \Pi_{Z}(F) \leq \Pi_{T}(F) \leq \Pi_{f}(F) .
$$

These inequalities tell us that $\Pi_{T}$ is dominated by $\Pi_{f}(F)$, but dominates strictly at least for one $F$, as seen above - the natural extension of $\Pi_{f}(F)$. Therefore $\Pi_{T}$ cannot be coherent: if it were so, this would contradict theorem 2 (c), applied to the natural extension $\bar{E}_{\Pi_{f}}(F)$.

Proof of (b): $\Pi_{T}$ dominates $\bar{E}_{\Pi_{f}}$, which (being coherent) avoids sure loss and therefore dominates some coherent precise prevision $P$ by theorem 1 (a); $\Pi_{T}$ then dominates $P$ and hence avoids sure loss, again by theorem 1 (a).

Proof of (c): let $\mathcal{M}\left(\Pi_{T}\right)\left(\mathcal{M}\left(\bar{E}_{\Pi_{f}}\right), \mathcal{M}\left(\Pi_{f}\right)\right)$ be the set of coherent precise previsions dominated by $\Pi_{T}$ (by $\bar{E}_{\Pi_{f}}$, by $\Pi_{f}$ ). From (16) and theorem 2,

$$
\mathcal{M}\left(\Pi_{f}\right)=\mathcal{M}\left(\bar{E}_{\Pi_{f}}\right) \subseteq \mathcal{M}\left(\Pi_{T}\right) \subseteq \mathcal{M}\left(\Pi_{f}\right),
$$

hence $\mathcal{M}\left(\Pi_{T}\right)=\mathcal{M}\left(\Pi_{f}\right)$, which means, applying again theorem 2 , that $\bar{E}_{\Pi_{T}}(F)=$ $\bar{E}_{\Pi_{f}}(F)$.

To complete the proof of the theorem, let now $\Pi_{T}$ be non-comparative. It is easy to see then that $\Pi_{Z}(F)=\Pi_{f}(F), \forall F \in \mathcal{F}$ (for instance, using (15)). Since applying the maximum operator over all $\omega \in \Omega$ to the members of (12) we get, $\forall F \in \mathcal{F}$,

$$
\Pi_{Z}(F) \leq \Pi_{T}(F) \leq \Pi_{f}(F)
$$

(incidentally, note that (18) holds also for a comparative $\Pi_{T}$ ), all $\mathrm{T}$-norms are equal to $\Pi_{f}$ in the non-comparative case. Coherence of $\Pi_{T}$ follows then from proposition 4 . 


\subsection{Discussion}

There are several points which deserve some discussion at this stage.

(a) Generalization to fuzzy T-seminorms. Although we presented the generalization (10) of (1) where min is replaced by a T-norm, theorem 4 still holds if min is more generally replaced by a $\mathrm{T}$-seminorm (definition 4 ). In fact, the key inequalities (12) apply also when $T(\pi(\omega), F(\omega))$ is a T-seminorm, as is easily verified using (a') and (e) of definition 4.

Further, the inequalities (18) hold too, $\forall F \in \mathcal{F}$ and for a given $\pi$. This means that replacing min with a $\mathrm{T}$-seminorm in (4) provides us with a better (while still not coherent, in the comparative case) evaluation, in the sense that it is closer to its least-committal coherent correction, the natural extension (11). In this view, the drastic product is preferable among all $\mathrm{T}$-seminorms.

(b) Fuzzy T-possibilities as fuzzy integrals. A fuzzy T-possibility is a way of extending a possibility (a special case of coherent upper probability) from $\mathcal{P}(\Omega)$ to $\mathcal{F}(\Omega)$. A fuzzy $\mathrm{T}$-possibility is also an instance of fuzzy integral, as defined by de Cooman $^{3}$ (generalizing Sugeno's original definition ${ }^{15}$; other generalizations include those by Garcia and Alvarez ${ }^{14}$ and Weber $^{21}$ ). According to this definition, given a bounded measurable non-negative map $X, X: \Omega \rightarrow \mathbb{R}^{+}$, a map $T: \mathbb{R}^{+} \times \mathbb{R}^{+} \rightarrow \mathbb{R}^{+}$ satisfying (d) and (e) of definition 4 and a possibility measure $\Pi$ on $\mathcal{P}(\Omega)$, the fuzzy integral of $X$ with respect to $\Pi$ is

$$
\text { (f) } \int X \mathrm{~d} \Pi=(f) \int_{\Omega} X \mathrm{~d} \Pi=\sup _{x \geq 0}\{T(x, \Pi(X \geq x))\} \text {. }
$$

When $T$ is the algebraic product, (19) reduces to the Shilkret integral ${ }^{13}$.

Under the assumptions of theorem 4,

$$
\text { (f) } \int F \mathrm{~d} \Pi=\max _{\omega \in \Omega}\{T(\pi(\omega), F(\omega))\},
$$

as follows from results by de Cooman and Kerre $^{5}$ or, more directly, from de Cooman $^{3}$, proof of eq. (10), recalling that any T-norm on a finite set is completely distributive with respect to supremum. Extensions of coherent upper probabilities and of possibilities using the fuzzy integral (19) are investigated by de Cooman ${ }^{2,}$, and our work therefore relates closely to these papers. In particular, part (a) of theorem 4 follows from general results in de $\operatorname{Cooman}^{2, ?}$; however, our proof is different and is instrumental for determining the natural extension $\bar{E}_{\Pi_{T}}$, a question which is not tackled in de Cooman ${ }^{2,}$.

We incidentally note also that the fact that when the given $\Pi$ is non-comparative all $\mathrm{T}$-seminorms coincide is again in accordance with a more general result in de Cooman $^{2, ?}$, stating that when a fuzzy integral is coherent, it is equal to the Shilkret integral.

(c) Properties of fuzzy $\boldsymbol{T}$-possibilities. An arbitrary upper prevision $\bar{P}$ which avoids sure loss but is not coherent may have some unpleasant features, like lack 
of internality or of monotonicity. Fuzzy T-possibilities avoid some of these shortcomings, in particular it is easy to verify that $\forall F \in \mathcal{F}, \Pi_{T}(F) \in[\min F, \max F]$ (internality) and, using isotonicity of T-(semi)norms, that if $F_{1} \leq F_{2}$ then $\Pi_{T}\left(F_{1}\right) \leq$ $\Pi_{T}\left(F_{2}\right)$ (monotonicity).

As for the closure properties of fuzzy T-possibilities, they are not guaranteed for every $\mathrm{T}$-norm, but continuity of $T$ is a sufficient condition for them to hold. This ensues from the next proposition and the subsequent example.

Proposition 7. (a) Let $\mathcal{P}_{\Gamma}=\left\{\Pi_{T \gamma}\right\}_{\gamma \in \Gamma}$ be a given family of fuzzy T-possibilities on $\mathcal{F}(\Omega)$, where $\Omega$ is arbitrary. If $T$ is continuous, the upper envelope $\Pi_{T}^{*}$ of $\mathcal{P}_{\Gamma}$, $\Pi_{T}^{*}(F)=\sup _{\gamma \in \Gamma}\left\{\Pi_{T \gamma}(F)\right\}, \forall F \in \mathcal{F}$, is a fuzzy T-possibility.

(b) Let $\Omega$ be finite and let $\left\{\Pi_{T}^{n}\right\}_{n \in \mathbb{N}^{+}}$be a sequence of fuzzy T-possibilities on $\mathcal{F}(\Omega)$, which converges pointwise to a function $\Pi_{T}$ on $\mathcal{F}$, i.e. $\Pi_{T}^{n}(F) \rightarrow \Pi_{T}(F), \forall F \in \mathcal{F}$. If $T$ is continuous, $\Pi_{T}$ is a fuzzy T-possibility.

Proof. The restriction $\left.\Pi_{T}\right|_{\mathcal{F}_{0,1}}$ of a fuzzy T-possibility $\Pi_{T}$ to the set $\mathcal{F}_{0,1}$ of all 0-1 valued $F \in \mathcal{F}$ is just an ordinary possibility. In fact, let $F \in \mathcal{F}_{0,1}$. When $F(\omega)=0$, $T(\pi(\omega), F(\omega))=0$ by (e) in definition 4, when $F(\omega)=1, T(\pi(\omega), F(\omega))=1$ by (a') in definition 4. Therefore $\Pi_{T}(F)=\max _{\omega \in \Omega}\{T(\pi(\omega), F(\omega))\}=\max _{\omega \in A}\{\pi(\omega)\}$, where $A$ is the event in $\mathcal{P}(\Omega)$ made up of those $\omega$ such that $F(\omega)=1$ (if $F \equiv 0$, $A=\emptyset$ and $\Pi_{T}(\emptyset)=0$ is assumed).

Proof of (a). From what just noted above and proposition $1, \pi^{*}(\omega)=\left.\Pi_{T}^{*}\right|_{\mathcal{F}_{0,1}}(\omega)$, $\forall \omega \in \Omega$, is a possibility $\left(\left.\Pi_{T}^{*}\right|_{\mathcal{F}_{0,1}}=\sup _{\gamma \in \Gamma}\left\{\left.\Pi_{T_{\gamma}}\right|_{\mathcal{F}_{0,1}}\right\}\right)$. The proof is then analogous to that of proposition 6 (a), using continuity of $T$ instead of continuity of min.

Proof of (b). Similarly to (a), note first that $\left.\Pi_{T}^{n}\right|_{\mathcal{F}_{0,1}}$ is a possibility, hence $\pi(\omega)=$ $\left.\lim _{n \rightarrow \infty} \Pi_{T}^{n}\right|_{\mathcal{F}_{0,1}}(\omega), \forall \omega \in \Omega$, is a possibility distribution by proposition 3 . The proof continues like that of proposition 6 (b) ( $\mathrm{min}$ is replaced by $T$ ).

\section{Example 1.}

The T-norm $Z$ (drastic product) recalled in (13) is clearly not continuous. Let now $\Omega=\left\{\omega_{1}, \ldots, \omega_{k}\right\}$, and $\left\{\Pi_{Z_{n}}\right\}$ be a sequence of fuzzy Z-possibilities, generated by the following distributions:

$$
\pi_{n}\left(\omega_{i}\right)=\left\{\begin{array}{cc}
1, & \text { if } i=1 \\
1-\frac{1}{n} & \text { otherwise }
\end{array}\right.
$$

Choose $F \in \mathcal{F}$ such that $0<F\left(\omega_{1}\right)<\max _{i=1, \ldots, k}\left\{F\left(\omega_{i}\right)\right\}<1$. Then

$$
\Pi_{Z_{n}}(F)=\max _{\omega_{i} \in \Omega}\left\{Z\left(\pi_{n}\left(\omega_{i}\right), F\left(\omega_{i}\right)\right)\right\}=F\left(\omega_{1}\right),
$$

and consequently $\Pi_{Z}(F)=\lim _{n \rightarrow \infty} \Pi_{Z_{n}}(F)=F\left(\omega_{1}\right)$.

From this, and since $\lim _{n \rightarrow \infty} \pi_{n}\left(\omega_{i}\right)=1, \forall \omega_{i} \in X$,

$$
\Pi_{Z}(F)=F\left(\omega_{1}\right)<\max _{\omega_{i} \in \Omega}\left\{F\left(\omega_{i}\right)\right\}=\max _{\omega_{i} \in \Omega}\left\{Z\left(\pi\left(\omega_{i}\right), F\left(\omega_{i}\right)\right)\right\} .
$$


This implies that proposition 7 (b) does not hold for the T-norm $Z$.

Nor does 7 (a) hold for $Z$, as follows from

$$
\begin{array}{r}
\Pi_{Z}^{*}(F)=\sup _{n \in \mathbb{N}} \max _{\omega_{i} \in \Omega}\left\{Z\left(\pi_{n}\left(\omega_{i}\right), F\left(\omega_{i}\right)\right)\right\}=F\left(\omega_{1}\right) \\
<\max _{\omega_{i} \in \Omega}\left\{F\left(\omega_{i}\right)\right\}=\max _{\omega_{i} \in \Omega}\left\{Z\left(\pi\left(\omega_{i}\right), F\left(\omega_{i}\right)\right)\right\} .
\end{array}
$$

\section{Conclusions}

Fuzzy possibilities may be seen as a way of extending ordinary possibilities to upper previsions, and may therefore be interpreted as upper previsions themselves. From this, it is natural to investigate their consistency properties within the wellestablished theory of imprecise probabilities (previsions). This kind of analysis seems necessary if we wish to give a behavioral explanation to the usage of tools from fuzzy set theory in a number of practical decision problems, like the one mentioned in the Introduction.

It turns out that fuzzy possibilities satisfy the weaker consistency requirement of avoiding sure loss, but generally not the stronger coherence condition, hence they are a weakly consistent model in this framework. However they preserve some closure properties of ordinary possibilities, and it is possible to correct them to coherent evaluations using the natural extension, which we determine explicitly.

It is also natural to generalize fuzzy possibilities replacing min with a $\mathrm{T}$ (semi)norm in their definition. This does not alter the consistency properties, and the natural extension remains the same too, while it appears that the closure properties hold when $T$ is continuous, not necessarily otherwise. Among all T-(semi)norms, the drastic product $Z$ is the closest to the natural extension, while min behaves in the opposite way. However we saw that the closure properties do not hold for $Z$. Hence no $\mathrm{T}-$-(semi)norm appears to be uniformly preferable, but any continuous one performs at least as well as min.

Finally, when viewing fuzzy $\mathrm{T}$-possibilities as instances of fuzzy integrals, this paper may be seen as a further contribution to prior work on the consistency of fuzzy integrals as imprecise previsions.

\section{References}

1. G. de Cooman (1997), Possibility theory I: the measure-- and integral-theoretic groundwork, International Journal of General Systems 25, 291-323.

2. G. de Cooman (2000), Integration in possibility theory, in Fuzzy Measures and Integrals: Theory and Applications (M. Grabisch, T. Murofushi and M. Sugeno eds.), 124-160, Physica-Verlag.

3. G. de Cooman (2001), Integration and conditioning in numerical possibility theory, Annals of Mathematics and Artificial Intelligence 32, 87-123.

4. G. de Cooman, D. Aeyels (1999), Supremum preserving upper probabilities, Information Sciences 118, 173-219.

5. G. de Cooman, E. E. Kerre (1996), Possibility and necessity integrals, Fuzzy Sets ans Systems 77, 207-227. 
6. L. Crisma (1990), Un approccio alla descrizione e valutazione dei numeri aleatori, in Scritti in omaggio a Luciano Daboni, Pubbl. n. 2 del Dip. Mat. Appl. "B. de Finetti", Edizioni Lint, Trieste.

7. B. de Finetti (1974), Theory of Probability, vol. I, Wiley, London.

8. D. Dubois, H. Prade (1988), Possibility Theory, Plenum Press, NY, USA.

9. Nguyen H. T., Walker E. A. (1998), A First Course in Fuzzy Logic, 2nd ed., Chapman \& Hall.

10. E. Miranda, G. de Cooman (2003), Epistemic indipendence in numerical possibility theory, International Journal of Approximate Reasoning 32, 23-42.

11. R. Pelessoni, P. Vicig (2003), Convex imprecise previsions, Reliable Computing 9 (6), 465-485.

12. W. Rudin (1976), Principles of Mathematical Analysis, 3rd ed., Mc-Graw-Hill.

13. N. Shilkret (1971), Maxitive measures and integration, Indag. Math., 33, 109-116.

14. F. Suarez Garcia, P. Gil Alvarez (1986), Two families of fuzzy integrals, Fuzzy Sets and Systems 18, 67-81.

15. M. Sugeno (1974), Theory of fuzzy integrals and its applications, Ph.D. Thesis, Tokyo Institute of Technology, Japan.

16. P. Walley (1991), Statistical Reasoning with Imprecise Probabilities, Chapman and Hall, London.

17. P. Walley (1996), Measures of uncertainty in expert systems, Artificial Intelligence 83, 1-58.

18. P. Walley (1997), Coherent upper and lower previsions, The Imprecise Probabilities Project, available at http://www.sipta.org/ documentation/ upper_lower_prev/ upper_lower_prev.html.

19. P. Walley, G. de Cooman (1999), Coherence of rules for defining conditional possibility, International Journal of Approximate Reasoning 21, 63-107.

20. Z. Wang, G. J. Klir (1992), Fuzzy Measure Theory, Plenum Press, NY, USA.

21. S. Weber (1986), Two integrals and some modified versions - critical remarks, Fuzzy Sets and Systems 20, 97-105

22. R. R. Yager, J. Fodor (1999), Fuzzy set-theoretic operators and quantifiers, in Fundamentals of Fuzzy Sets (D. Dubois and H. Prade eds.), Kluwer Academic Publishers, $125-193$.

23. L. A. Zadeh (1968), Probability measures of fuzzy events, J. Math. Anal. Appl. 23, 421-427.

24. L. A. Zadeh (1978), Fuzzy sets as a basis for a theory of possibility, Fuzzy Sets and Systems 1, 3-28. 